\title{
EVALUATION OF YARN LATERAL DEFORMATION
}

\author{
Krupincová G. ${ }^{1}$, Drašarová J. ${ }^{2}$, Mertová I. ${ }^{1}$ \\ ${ }^{1}$ Technical University of Liberec, Department of Textile Technology ${ }^{2}$ Technical University of Liberec, Department of Textile Design \\ Studentská 2, 46117 Liberec 1, Czech Republic, Tel.: +420 48535 3424, Fax: +420 485353542 \\ E-mail: gabriela.krupincova@tul.cz, jana.drasarova@tul.cz, iva.mertova@tul.cz
}

\begin{abstract}
:
The article focuses on a new approach for characterization and evaluation of lateral yarn deformation. A small review about theoretical description and measurement possibilities will be introduced. The evaluation of yarn compression will be done by three innovative methods (lateral deformation of yarn between two parallel plates, simulation of binding point of fabric, cross-sectional analysis of real fabric). The analysis of yarn deformation will be carried out for a set of samples in combination of fiber material, yarn count and given fabric structure.
\end{abstract}

\section{Keywords:}

Yarn diameter, lateral deformation of yarn, innovative measurement principals.

\section{Introduction to fabric structure}

The woven fabrics structure is complicated due to its complex hierarchy. There are no models, which are able to describe the structure from the fibers through the yarns, and to the fabric. Usually the yarn is used as an elementary building unit of the structural model of the fabric. The fineness $T$, twist $Z$ and diameter $d$ are the basic geometrical characteristics describing a yarn. The diameter is considered only as a theoretical idea. For evaluation of the yarn diameter, it is necessary to know the packing density $\mu[1,9]$.

The simplified assumption, that the yarn is compact, solid and circular cross-section, is implemented for a description of binding point geometry. The troubles with establishing the yarn diameter originate in the incompactness of yarn structure. Some air gaps are found between the fibers; the yarn crosssection (especially in binding point) also is not a circle. In the binding points, the deformation of the cross-section and the compression of fibers are considered.

In the stretched state of fabrics, yarns compress each other at their cross-over points. The lateral compression force at the cross-over points is generated by the yarn tension. Internal tension in fabric structure is given by the balance of forces, which depends on different types and levels of deformation during fabric production stages and its use. The typical deformation of the yarn cross-section is generally caused by the combined effect of compression, extension, bending and torsion.

Main aim of this work is to report about possibilities, which can be used for measuring the lateral deformation of yarn. The evaluation of yarn compression will be done by three innovative methods (lateral deformation of yarn between two parallel plates, simulation of binding point of fabric, crosssectional analysis of real fabric). The experimental results for the selected material, yarn count and fabric structure will be presented.

\section{Yarn cross-section deformation}

The nearly circular yarn cross-section is due to change of compression to a more flat profile. A lot of geometrical models do not include this phenomenon and the so-called free yarn geometry is assumed. Yarn flattening is important from the point of view of selected fabric parameters evaluation, modeling and design. It is, for example, fabric porosity, air permeability and mechanical characteristics in terms of ultimate and user range loading.

\subsection{Models}

The width $a$ and height $b$ are defined for the description of yarn cross-section deformation. The circular cross-section of the "free" yarn (Figure 1a) is changed into a shape, which can be substituted by Kemp's cross-section (oval of two halfcircles with semi-diameter $b$ and two abscissas of length $a-b$, Figure 1b), elliptical or lens shape (Figure 1c, d) [1].
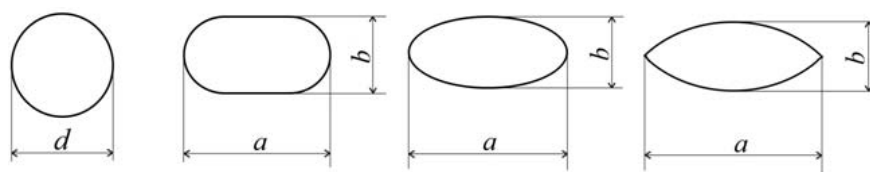

Figure 1. Shape of yarn cross-section:

a) "free" yarn, b) Kemp, c) ellipse, d) lens.

It is possible to define relative enlargement and relative compression:

$$
\begin{aligned}
& \varepsilon_{1}=(b-d) / d \\
& \varepsilon_{2}=(a-d) / d
\end{aligned}
$$

The area $S$ and the perimeter $L$ of these shapes can be easily calculated. 


\section{$\underline{2.2 \text { Geometrical hypothesis }}$}

For the relationship between the shapes, two alternative hypotheses about constant area and constant perimeter are proposed. This hypothesis can be described as a function of relative enlargement $e_{1}$ and relative compression $e_{2}$.

Constant area (cross-sections before and after deformation have the same area) subsequently holds:

$$
S=\frac{\pi d}{4}=S_{\text {deformed yarn }}
$$

The relation between relative enlargement and relative compression is derived:

$$
\begin{array}{ll}
\text { Kemp } & \varepsilon_{2}=\left(\varepsilon_{1}^{2}(1-\pi / 4)+\varepsilon_{1}(1-\pi / 2)\right) /\left(\varepsilon_{1}+1\right), \\
\text { ellipse } & \varepsilon_{2}=-\varepsilon_{1} /\left(\varepsilon_{1}+1\right)
\end{array}
$$

It is impossible to express the relative enlargement explicitly for lens; therefore, the equation was solved numerically:

$$
\text { lens } \varepsilon_{2}=1,11 /\left(\varepsilon_{1}+1\right)^{1,06}-1 \text {. }
$$

Constant perimeter (cross-sections before and after deformation have the same perimeter) subsequently holds:

$$
L=\pi d=L_{\text {deformed yarn }} .
$$

The relation between relative enlargement and relative compression is derived:

$$
\begin{array}{lll}
\text { Kemp } & \varepsilon_{2}=\varepsilon_{1}(1-\pi / 2), \\
\text { ellipse } & \varepsilon_{2}=\sqrt{2-\left(\varepsilon_{1}+1\right)^{2}}-1 \\
\text { lens } & \varepsilon_{2}=\sqrt{(\pi / 2)^{2}-4 / 3\left(\varepsilon_{1}+1\right)^{2}}-1 .
\end{array}
$$

Lomov [7] proposes the relation between relative enlargement and relative compression empirically:

$$
\varepsilon_{2}=1 /\left(\varepsilon_{1}+1\right)^{n}-1 \text { for }(n=1,2 \ldots) \text {. }
$$

The estimation of these hypotheses is based on the assumption of circular cross-section of "free" yarn. The circle has minimal perimeter for the same area and maximal area for the same perimeter, compared to other plane figures. The cross-section changes are caused not only by changes in shape but also due to relaxation of radial forces. These forces originate from the helix structure of the fibers in the yarn.

From the first hypothesis of "constant area", we conclude that the perimeter of the deformed cross-section must be increasing; the volume of inter-fibers pores does not change, it means that the packing density decreases. It would be a particular effect, which eliminates the action of radial forces relaxation.

From the second hypothesis of "constant" perimeter, the area of the deformed cross-section must be decreasing; the packing density increases, because the number of inter- fiber pores decreases and the contacts of fibers increase. It means the destruction of the original (primary) yarn structure turn up.

\section{Experimental methods}

There exist various methodologies for evaluation changes in yarn cross-sections. A change of yarn diameter under tension in the yarn-axis direction was studied in many pieces of research. They are mostly based on optical system or mechanical detection. Only few of them are described and their results are shown in this article.

There is a group of methodologies, which is based on fabric analysis. One of them is the judgment of fabric thickness before and after biaxial tension, which is described in [4]. The crosssectional analysis of fabric in freeze state is another approach to gain information about yarn's deformation. Fabric can be fixed by soft or hard methods. The experiment is based on the analysis of frozen fabric structure in terms of cross-sectional analysis or surface analysis in the third main fabric direction [5]. The improved possibility of novel stress-freezing technique for studying the compression behavior of fabrics is described in [10]. The biggest advantage of this modified method is the possibility of deformed fabric fixing.

Fixing of fabric in stressed state is limited and therefore the simplified approaches were found to see the influence of selected factors and forces. Methodologies, which take not only compression but also bending into consideration, are wire method, $\mathrm{V}$ wire method, three-rod unit and simulation of binding point in hollow block [2] and [4].

The deformation of yarn between two parallel plates is the highest simplification of a real state in balance of forces at a cross-over point. In this case, only complexional forces cause the deformation of yarn. There are several methods by which the thickness of yarn can be measured. Using of a rotation drum and feeler, in which the yarn thickness is measured by passing the yarn around the drum's circumference with the feeler pressed very gently against the yarn, has been mentioned in [8]. The KES F3 system allows the measurement of yarn compression in terms of yarn thickness as a function of compression load $[3,4]$. In case, the information about yarn thickness (minor yarn diameter $b$ ) is not enough and the knowledge of yarn widening (major yarn diameter $a$ ) is demanded then it is possible to use special equipments, which offer measuring the change in yarn diameter in both main directions under higher pressure [2].

\subsection{Analysis of weave cross-sections}

The method used for the detection of the internal weave structure is based on the analysis of the weave cross-sections $[4,5,7]$. The measuring parameters are shown in Figure 2 . The fabric cross-sections were prepared by the method of "soft" cross-sections, where the blend of bee wax and paraffin, as fixing medium, was used $[1,9]$. These cross-sections usually have a thickness of $30 \mathrm{~mm}$. 


\subsection{Assessment of yarn flattening caused by compression and bending}

The wire and $V$ wire method is the simplification of real state in fabric binding point. One of the cross-over yarns is substituted by absolute stiff wire because of the factors influencing elimination. A steal wire is usually fixed on a horizontal base and yarn is hanged on the wire with tension. The angle between the yarn and the horizontal level is $30^{\circ}$, which is approximately equal to the averaged yarn-intersecting angle in various weaves. A V-shaped groove at the cross-over area is more close to the real intersecting state of the yarn in fabric binding point. The yarn thickness is measured by a needle sensor contacting at the top of the yarn surface with a small compression force $[4,9]$.

The alternative possibility based on the $V$ wire method is using the three-rod unit mounted, for example, on the Instron Tensile Tester. The three-rod unit consists of a rod fixed to a horizontal base and two rods of the same diameter placed parallel to the bottom one. It is spaced at the center of the three rods that form an equilateral triangle. The unit enables the measurement to be made of the changes in both minor and major diameters of a yarn bent over the three-rod unit subjected to increasing extension [4].

Simulation methodology of real fabric binding points goes out from idea that the yarns are crossed in a hollow block and various forces realize their deformation. Arrangement of experiment is shown in Figure 3. The hollow block is placed under a macroscope and the measurement of change in yarn's diameters is realized in the system of image analysis [2]. Yarn is guided in between two opposite corners that are placed in position of block diagonals. One end of the yarn is fixed by clumps and the other is guided over a small ideal pulley with a small pretension. The simulated binding point is placed in the hollow block center. The loading of the yarn sample is realized by various weights.

\subsection{Yarn compression between two parallel plates}

The other method for the simulation of stress in the binding point is based on the yarn compression between two parallel plates $[5,6]$. The first prototype is shown in Figure 4. This device is placed under a macroscope holder. The yarn is guided though the measuring zone between two glass parallel plates and is pretended proportionally to the yarn count. The deformation of yarn is the result of loading realized by an upper frame. The upper frame can be connected with various pieces of defined weight and a seven level of loading is available. Sequence of yarn longitudinal views before and after deformation at same place is scanned and the absolute values on scales of two contact thickness meters are read. Sequences of yarn macro-images before and after deformation in terms of yarn diameter $\mathrm{d}$ and yarn characteristic proportion a are evaluated. Characteristic proportion $b$, in other words yarn thickness, after deformation is equal to the value, which describes the difference between the absolute value on the scale of thickness meter without and with deformed yarn between parallel plates under pressure.

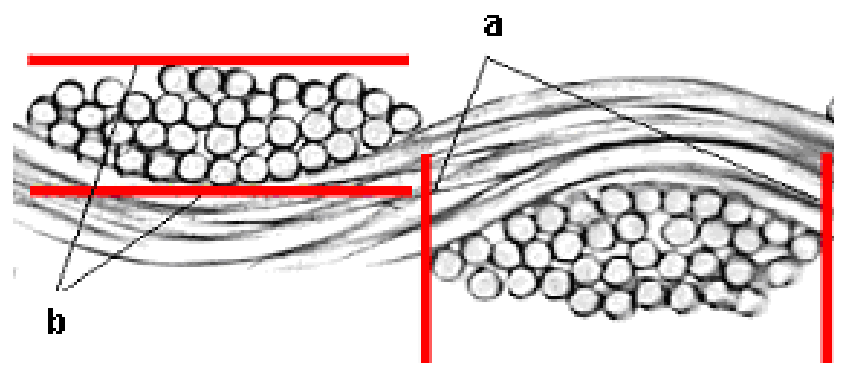

Figure 2. Measured values.

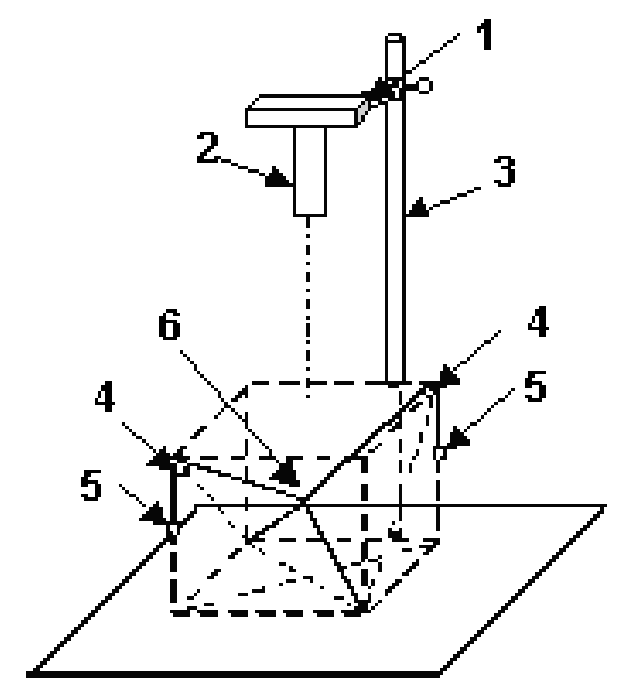

Figure 3. Hollow block:

1 camera
2 macro-scope objective
3 tripod
4 pulley
5 weight
6 simulated binding point
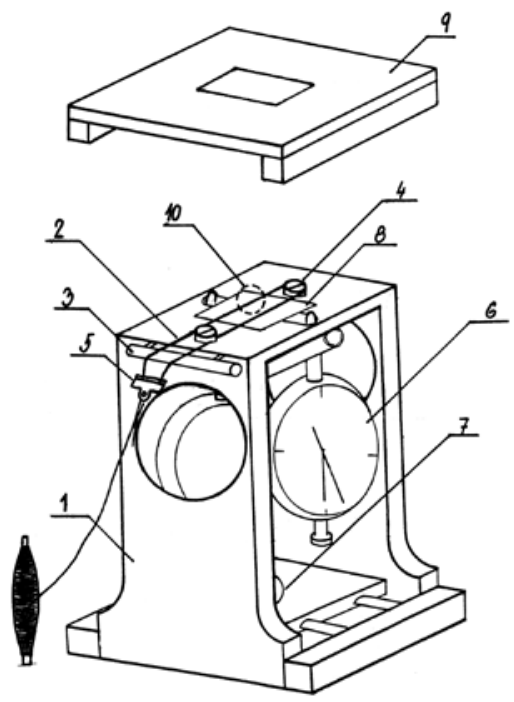

Figure 4. Measuring device for yarn compression:
1 main frame
2 yarn
3 guide
4 brake
5 straightening load
6 contact thickness meter
7 lighting system
8 lower plate with calibrated ruler
9 frame with upper glass plate
10 measuring area 


\section{Experimental material and results}

The main idea of this experiment was to use the selected methodologies used for the evaluation of yarn deformation and apply them to a set of fabrics and yarns, which were used for fabric weaving. The results should be discussed and compared with expectations and the influencing factors should be identified.

A set of experimental gray relaxed fabric in plain weave was used for the experiment. Fabrics were produced from $100 \% \mathrm{CO}$, $100 \%$ PP and 50CO/50 PP 29,5tex staple single ring yarns with a given set of warp ( 25 thread $\mathrm{cm}^{-1}$ ) and three level of set of weft (8,8 thread $\mathrm{cm}^{-1}$, optimum 13 thread $\mathrm{cm}^{-1}$ and 17 thread $\mathrm{cm}^{-1}$ ). One gray relaxed fabric produced with comparable geometrical structure in plain weave from 100\%PET 16,5tex staple single yarn was added for the experiment. Analysis of yarn deformation was realized according to the cross-sectional technique described in section 3.1 for both main directions of the fabric (warp and weft). Experimental results are shown in Figure 5a.

Estimation of the level of yarn deformation based on the evaluation of simulated binding point described in section 3.2 was realized for a set of yarn used for fabric production. Yarns were spun by classical ring spinning technology with 29,5tex yarn count from $100 \%$ CO, 100\% PP and 50CO/ 50 PP staple fiber material. Three levels of loading force were applied to simulate yarn deformation $(1,8 \mathrm{~g}-0,07 \mathrm{~N}, 6,8 \mathrm{~g}-0,26 \mathrm{~N}$ and $11,8 \mathrm{~g}-0,44 \mathrm{~N})$. The obtained data are presented in Figure $5 \mathrm{~b}$.

Yarn deformation was also simulated by deformation between two plates mentioned in section 3.3. Hundred percent $\mathrm{CO}$ 29,5 tex staple single ring spun yarn, which was used for fabric production, was analyzed and an experimental set of $100 \%$ CO single staple yarn was added to the lab measurements. It was a set of typical ring spun yarn that was produced with 16,5 tex, 20 tex and 38tex yarn count. Seven levels of deformed forces were used for the simulation of yarn deformation $(10 \mathrm{~N}, 15 \mathrm{~N}$, $20 \mathrm{~N}, 25 \mathrm{~N}, 30 \mathrm{~N}$ and $40 \mathrm{~N}$ ). Summarization of data is given in Figure 5c.

\section{Discussion}

Figures 5 a, b, c show relationships between relative enlargement and relative compression. The hypothesis for constant perimeter and constant area are compared with experimental data.

The generally known results could be expected. Experimentally analyzed yarn deformations in a fabric binding point are located in the low levels of relative yarn enlargement and compression. It is not possible to decide, if the deformations follow the hypothesis of constant perimeter or constant area. Calculated curves describe that the limited cases are very close to each other in this range of deformations. Experimental data should be placed in a delimited area of both hypotheses. Most of them are under hypothesis of constant perimeter. It is probably caused by the precision of original yarn diameter estimation.

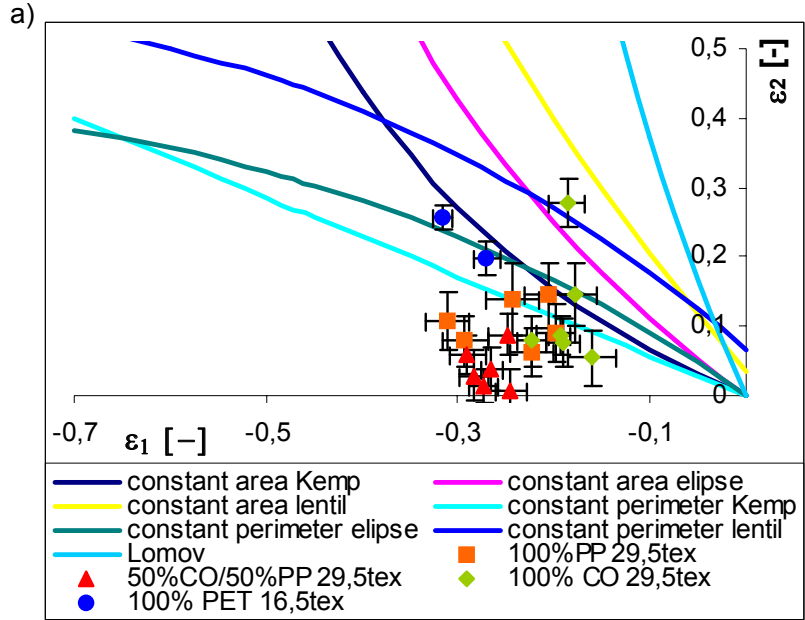

b)

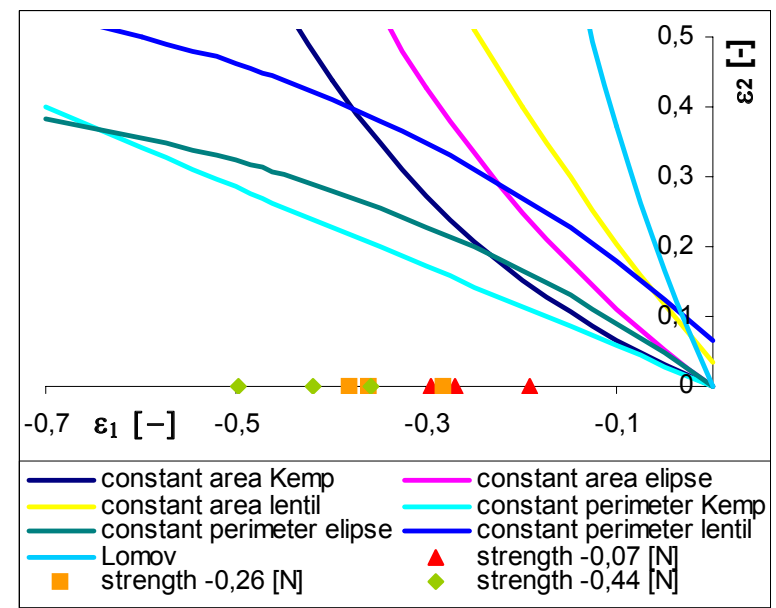

c)

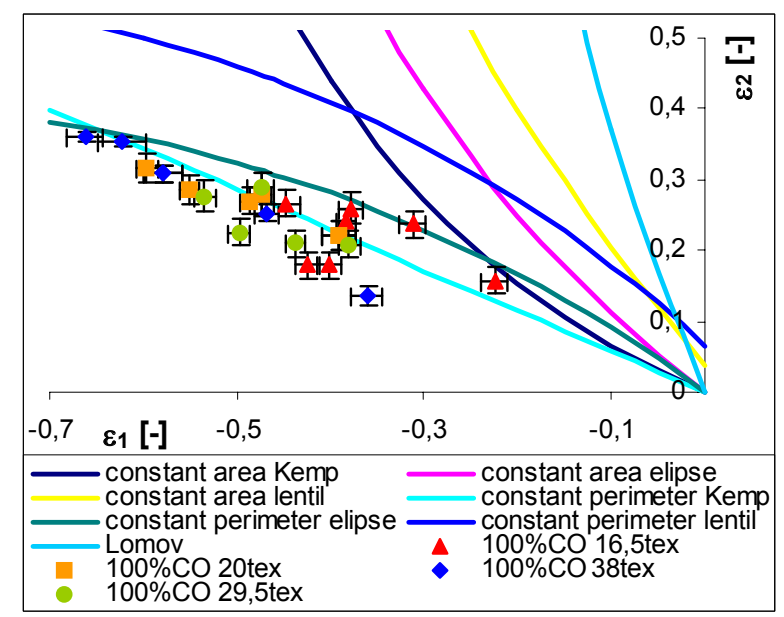

Figure 5. Experimental results:
a) method 3.1
b) method 3.2
c) method 3.3

The influence of evaluated factors was in accordance with previous experience. Higher deformations in terms of relative enlargement and compression were found for the main fabric warp dimension. From the point of view of weft analysis, it can be said that the deformation increases when the weft set 
increases. The behavior of fabric produced from blended yarn is more close to the behavior of $100 \%$ PP fabric. Differences among experimental data were very small. The verification of significance power of these factors is limited, because the data are very sensitive to original yarn determination.

Using of hollow block for the analysis of yarn deformation is limited from the point of view of estimation of relative compression only. In other words, it is not possible to measure the second parameter describing the level of relative enlargement. Therefore, the second coordinate of the experimental data is equal to zero in Figure $5 \mathrm{~b}$. An increased loading force causes the increase of yarn relative compression in contact point. The statistical significance of the fiber material used for yarn production is very low but for verification repeating of measurements should be realized. The blended yarn behavior is much closer to the behavior of $100 \% \mathrm{PP}$ yarn. The reason can be what's hidden in yarn production because of used mass fibers mixing. There is a higher number of polypropylene fibers in yarn volume than cotton fibers. It confirms the outputs from the methodology given in section 3.1. The interesting conclusion arises from the comparison of relative deformation obtained form fabric analysis and this method. It seems that the level of normal force in the gray relaxed fabric is very close to the first level of loading force realized here by the weight $1.8 \mathrm{~g}$.

Simulation of yarn between two parallel plates is the most simplified process of real deformation in real binding point. Based on previous experiments and solving force balance, it can be expected that for the description of deformed yarn the cross-section Kemp model and hypothesis of constant perimeter will be desirable. It means, in other words, that the yarn is due to deformation compressed and the pacing density of yarn increases. It can be also expected, that the deformation will increase with the increase in yarn count or loading force. The obtained experimental results are in a good agreement with our expectations. Differences in experimental data results are higher for the higher applied forces. Moreover, the statistical significance of data differences was not verified.

\section{Conclusion}

In this investigation, the yarn lateral deformation was studied. The experimental data obtained from three selected methodologies were compared. The influence of selected factors was roughly evaluated. There was for analysis used methodology, fiber material, yarn count, applied level of deformation force and fabric structure in terms of set of warp and weft.

It can be concluded (thanks to the realized experiment) that the methodologies give us comparable results, which can be the background for precise modeling of structure and mechanical parameters of fabric. Yarn flattening is important, for example, for estimation of fabric porosity, air permeability and mechanical characteristics in terms of ultimate and user range loading.

\section{Acknowledgment}

This work was supported by the research project GACR 106/09/1916.

\section{References}

[1] Drašarová, J.: Analysis of fabric cross-sections. PhD. Thesis, FT TUL, Liberec 2004, in Czech.

[2] Drašarová, J., Jamborová, J., Dzurindak, P.: Lateral deformation of yarn. FRVŠ 2000 2001, in Czech.

[3] Göktepe, E., Lawrence, C. A.: Deformation of Yarn Crosssection in Relation to Yarn Structure. Fibers \& Textile in Eastern Europe. April/ June Vol. 2 No. 33, 2001, ISSN: 1230-3666.

[4] Kawabata, S., Niwa M., Matsudaria, M.: Measurement of Yarn Thickness Change Caused by Tension and Lateral Pressure by Wire Method. Journal of Textile Machinery Society of Japan, Vol. 31, No. 1, 1985, ISSN: 1883-8723.

[5] Křemenáková, D., et all: Internal Standards. Textile Research Center, Technical University of Liberec 2003.

[6] Křemenáková, D., Krupincová, G.: Lateral compression of free yarn. International conference ARCHTEX 2003, ISBN 8391780805 .

[7] Lomov, S.V., Peeters, T.: Integrated textile preprocessor WiseTex. Version 2.3. Specific software User's guide.

[8] Mahmoudi, M. R., Oxenham, W.: A new electro-mechanical method for measuring yarn thickness. Autex Research journal, Vol. 2., No. 1, March 2002, ISSN: 1470-9589.

[9] Neckár̆, B.: Yarn. Creation, structure, properties. SNTL Praha 1990, ISBN: 80-03-00213-3, in Czech.

[10] Potluri, P., Wilding, M. A. and Memon A.: A Novel stressFreezing Technique for Studying and Compressional Behavior of Woven Fabrics. Textile Research Journal, Vol 72, No. 12, Dezember 2001, ISSN: 00405175. 